\section{Cureus}

\title{
Leiomyosarcoma Involving the Sciatic Nerve: Case Report and Review of the Literature
}

\author{
Judith Murovic $^{1}$, Anand Veeravagu ${ }^{1}$, David B . Bingham ${ }^{2}$, John K. Ratliff ${ }^{1}$, David G. Mohler ${ }^{3}$ \\ 1. Department of Neurosurgery, Stanford University School of Medicine 2. Department of Pathology, \\ Stanford University Medical Center 3. Department of Orthopedic Surgery, Stanford University School of \\ Medicine
}

$\square$ Corresponding author: Judith Murovic, jamurovic@yahoo.com

Disclosures can be found in Additional Information at the end of the article

\section{Abstract}

Soft tissue sarcomas (STSs) represent $1 \%$ and $15 \%$ of adult and pediatric tumors, respectively, and almost $75 \%$ of all sarcomas; primary STSs occur commonly in the lower extremities. Leiomyosarcomas originate from the malignant transformation of a smooth muscle cell, which in turn may arise from a pluripotent mesenchymal stem cell.

The present case report describes a patient with a leiomyosarcoma arising from the posterior thigh musculature, which invaded all layers of the left sciatic nerve's tibial division. Only two similar case reports exist in the literature, documenting sciatic nerve involvement by a leiomyosarcoma.

The current patient underwent preoperative fractionated external beam radiation therapy (FEBRT) to the tumor and then had its removal which resulted in a $12 \mathrm{~cm}$ resection of the tibial division of the sciatic nerve. This defect was repaired with an intrafascicular sural nerve graft. Postoperatively, she had weakness of left hip flexion, dorsi- greater than plantar flexion, and left eversion, all of which reverted to normal after approximately two years.

After surgery, the patient received further FEBRT, developed pulmonary involvement by leiomyosarcoma and underwent bilateral thoracotomies with removal of all nodules. She underwent chemotherapy (CTX) with ifosfamide and doxorubicine, the latter switched to epirubicin, but developed a new pulmonary nodule five months after CTX completion. Other CTX trials were unsuccessful, and she succumbed five years after her original extremity tumor complete resection.

Two other reported cases of leiomyosarcoma involving the sciatic nerve were found. One tumor arose from, and the other involved, the nerve, the latter as did ours and are reviewed. Sciatic nerve anatomy, clinical presentation and examination are presented, along with sarcoma

Published 06/07/2012

\section{(c) Copyright 2012}

Murovic et al. This is an open access article distributed under the terms of the Creative Commons Attribution License CC-BY 3.0., which permits unrestricted use, distribution, and reproduction in any medium, provided the original author and source are credited. genetics and molecular pathways involved in sarcomagenesis. A study on sarcoma invasion along a peripheral nerve trunk is reviewed, and the perineural space microenvironment of growth advantage, which enables tumor cell proliferation and spread, is described. Sarcoma chemotherapy and recurrence rates are summarized in the setting of the patient's chemotherapy.

Categories: Pathology, Neurosurgery, Orthopedics

Keywords: ifosfamide, fractionated external beam radiation therapy, doxorubicin, gemcitabine, 


\section{Introduction}

Soft tissue sarcomas (STSs) and primary bone sarcomas are the two main categories of sarcomas accounting for $1 \%$ and $15 \%$ of all adult and pediatric tumors, respectively [1]. Soft tissue sarcomas represent approximately 75\% of all sarcomas; the remainder are bone sarcomas [2-3]. Primary STSs most commonly occur in the lower extremities (46\%), specifically in the groin, buttock, and thigh regions. The remainder present in the retroperitoneum, head and neck, torso, and upper extremities $[2,4]$.

Leiomyosarcoma is an STS originating from the malignant transformation of a smooth muscle cell. Smooth muscle cells, in turn, may arise from a pluripotent mesenchymal stem cell [5].

The present case report depicts a patient with a leiomyosarcoma arising from the posterior thigh musculature which invaded all layers of the left tibial division of the sciatic nerve. This is a highly unusual tumor involving the sciatic nerve with only two previous reports in the literature [6-7].

\section{Case Presentation}

A 55-year-old female presented in November of 2005 with a one-month history of a painful left posterior thigh mass which was increasing in size. The patient described cramping sensations in the left lateral thigh with a tingling sensation in the left medial calf which extended into the first and second toes. Neurological examination at presentation revealed a left posterior thigh mass which, upon palpation, elicited tingling in the left first toe. Bilateral lower extremity motor and sensory examinations were found to be intact.

Magnetic resonance imaging (MRI) showed a mass which measured $8 \times 10 \mathrm{~cm}$ in the axial and cranio-caudal planes, respectively, and involved the left semi-tendinosus, biceps femoris, and possibly the abductor magnus muscle and the neurovascular structures in this region (Figure 1). The mass was further noted to extend along the anterior surface of the sciatic nerve. Per the neuroradiological report, the sciatic nerve was considered to be less likely the origin of this mass, but rather was encased by this mass. There was extensive T2 hyperintensity extending superiorly and inferiorly relative to the mass. The popliteal vessels distal to the adductor hiatus were not encased, and there was no significant adenopathy. 


\section{Cureus}
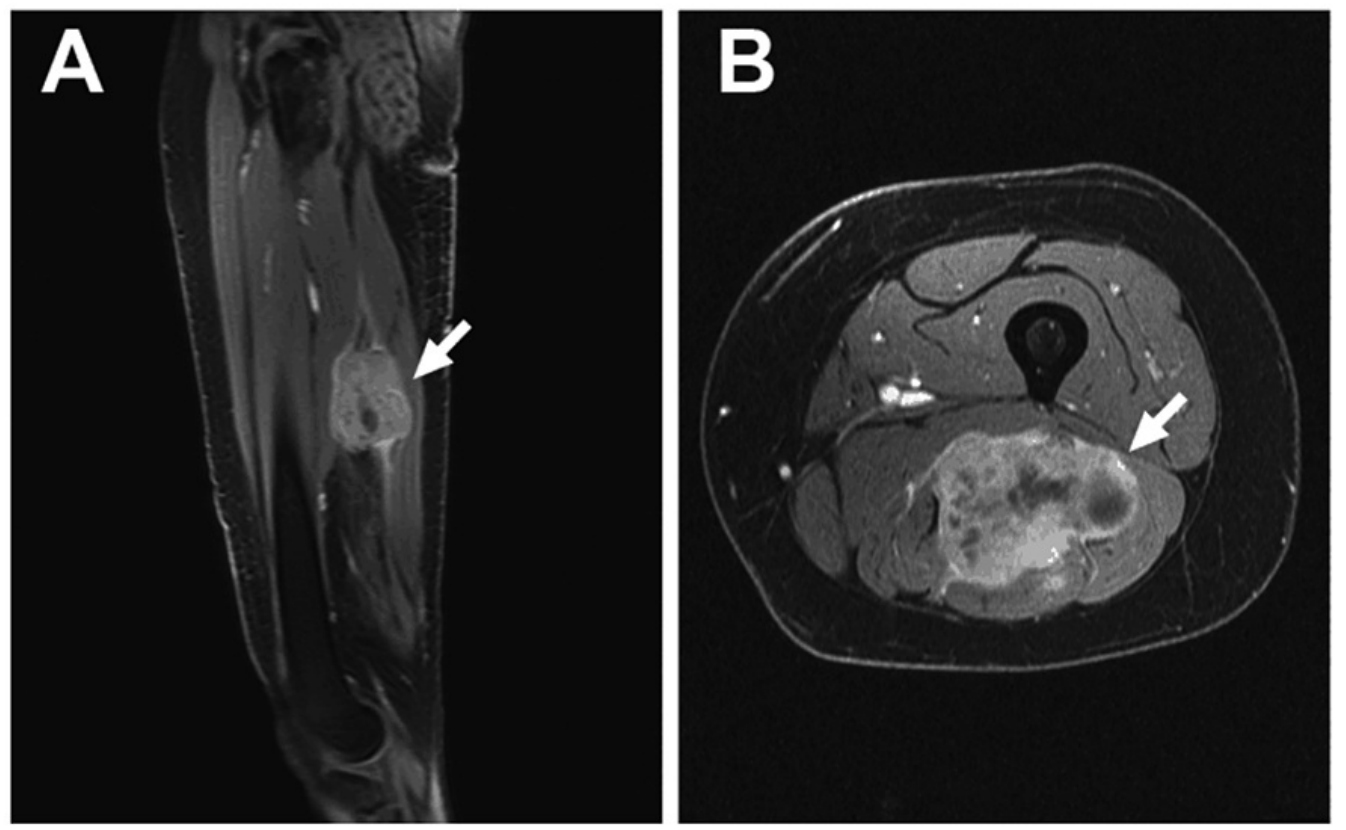

\section{FIGURE 1: The popliteal vessels distal to the adductor hiatus were not encased and there was no significant adenopathy}

The popliteal vessels distal to the adductor hiatus were not encased and there was no significant adenopathy. Figures $1 \mathrm{~A}$ (coronal) and $\mathrm{B}$ (axial) magnetic resonance imaging (MRI)slices above show the current case report patient's leiomyosarcoma, which involved the sciatic nerve's tibial division. The MRI was carried out using a post-contrast fat-suppressed spoiled gradient refocused acquisition in the steady-state.

A percutaneous biopsy of the mass in early December of 2005 revealed a leiomyosarcoma (Figure 2). Histopathological manifestations included spindle cell proliferation with marked nuclear proliferation and an irregular coarse chromatin with occasional prominent nucleoli; the mitotic count was 10 to 11 per high power field, and there were atypical mitotic figures seen. The immunohistochemical stains showed the tumor cells to be positive for actin and negative for CKMIX, S100, desmin, and CD 34.

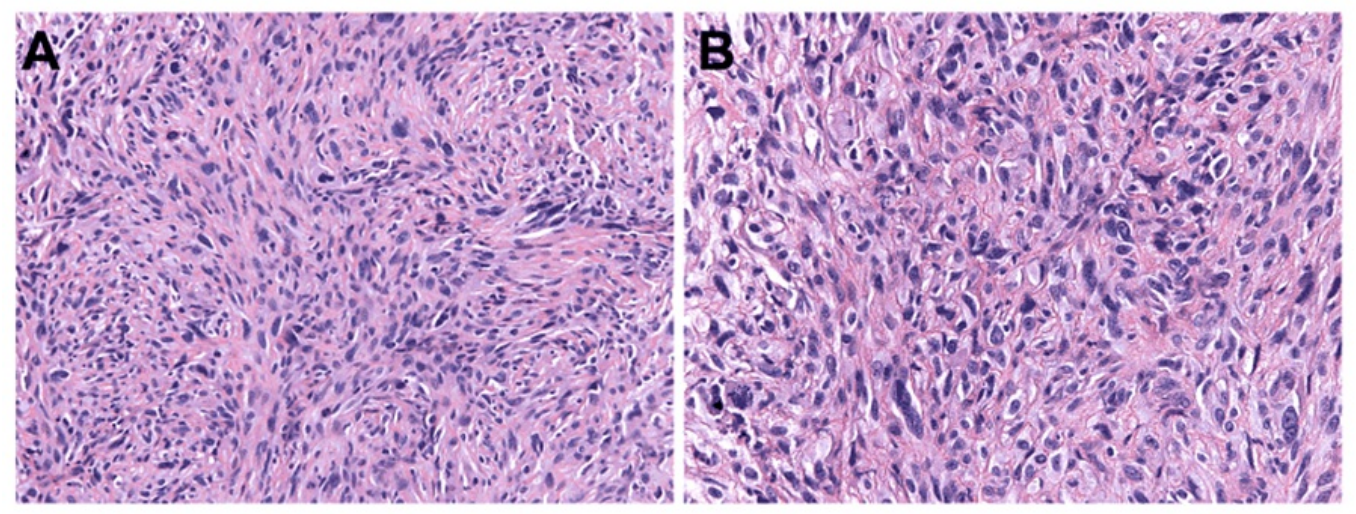

\section{FIGURE 2: Histologic sections}

Histologic sections show, on the left, low magnification (350 xs) and on the right, higher magnification (400 xs), a malignant spindle cell lesion with prominent nuclear pleomorphism and numerous mitotic figures. 
In an attempt to preoperatively reduce tumor burden away from the sciatic nerve, the patient underwent fractionated external beam radiotherapy (FEBRT). This was administered from December of 2005 through February of 2006 during which time she received $5000 \mathrm{cGy}$ to an area from the sciatic notch to the proximal lower leg because of significant MRI changes within the sciatic nerve.

In March of 2006, the patient underwent a radical resection of the tumor. The peroneal portion of the sciatic nerve was uninvolved by tumor; the tibial division, however, was adherent to and directly involved by the tumor and could not be separated from it for $9 \mathrm{~cm}$. Removal of the involved tibial section created a $12 \mathrm{~cm}$ nerve defect, the ends of which were anastomosed using an intrafascicular sural nerve graft. The tumor histopathology report stated that more than $99 \%$ of the tumor was necrotic.

The patient's postoperative neurological examination revealed motor strength in the lower extremities to be $5 / 5$, with the exceptions of a left hip flexor weakness of 3-/5; left dorsiflexion and extensor hallucis longus function were $3 / 5$. Left plantar flexion and eversion were $2 / 5$. Her sensory examination demonstrated a decrease to pinprick and light touch in the left anterior thigh and the sole of her left foot. The deep tendon reflexes were $2+$ at the right and left knees and $2+$ at the right and $1+$ at the left ankles.

Postoperatively, she underwent further FEBRT to the left thigh from April to May of 2006. The dosage consisted of $1440 \mathrm{cGy}$.

The patient developed several bilateral small pulmonary nodules seen on computerized tomographic (CT) scan in May of 2006. A CT scan one month later showed an increase in size of the mentioned nodules and the development of two new nodules. She underwent bilateral thoracotomies and wedge resections of the pulmonary nodules in August of 2006 and the reports documented that all lesions were consistent with leiomyosarcoma.

She was started on one course of ifosfamide and doxorubicin (Adriamycin) in September of 2006, and after this course, doxorubicin (Adriamycin) was switched to epirubicin to enable outpatient treatment. She completed the third through fifth courses in December of 2006. A right $3 \mathrm{~mm}$ apical nodule appeared on a chest CT five months post-completion of her chemotherapy, and this lesion remained quiescent.

Neurological examination in February of 2008 revealed complete return of the motor function of her left lower extremity muscles. She remained with residual numbness of the sole of her left foot.

Between March and August of 2008 she developed new bilateral lung lesions, as well as liver and retroperitoneal nodules. The two largest lung lesions were treated with CyberKnife radiosurgery administered in November of 2008: a right lower lung lesion was treated to 2500 cGy and a right anterior lower lobe target underwent $2923 \mathrm{cGy}$. Two new lesions in the peripheral right lower lobe enlarged, and she underwent Trilogy treatment of a right lower lobe lateral and an anterior lesion in June of 2009.

She was started on Gemzar (Gemcitabine Hcl) and Taxotere (Docetaxel) given together every two weeks from November of 2009 to March of 2010 at which time she received cycle 5B. The CT scans of the chest, abdomen and pelvis in April of 2010 compared with those from October and 
December of 2009 showed progression of her lung lesions, which trabectedin (Yondelis) administered in September of 2010 did not stop. She was then started on Bevacizumab (Avastin) and Temodar (Temozolamide) in November of 2010, which was complicated by severe cytopenias, and she remained off all therapy after this. A painful right posterior chest wall mass was treated by FEBRT to a total dose of $30 \mathrm{~Gy}$.

She had a chest MRI in May of 2011 which showed left axillary and large left and right pleuralbased masses, the right extending into the diaphragm and right retroperitoneal space and invading the T10-T12 central spinal canal with associated thecal sac compression; multiple parenchymal metastases were noted. She expired in June of 2011 in a hospice facility.

\section{Discussion}

A literature search revealed only one other similar case reported by Benyahya, et al. [6]. Their patient was a 22-year-old female who developed a leiomyosarcoma of the right buttock which infiltrated the right sciatic nerve. In this case, the right buttock leiomyosarcoma invaded the right piriformis, obturator internus, gemelli, and gluteus medius muscles, and a portion of the tumor extended into the pelvic cavity via the right sciatic notch. The tumor which invaded the right sciatic nerve was only able to be partially excised. Fractionated external beam radiotherapy was given postoperatively, and per the authors, "the patient's health deteriorated" and she was lost to follow-up. This tumor caused right leg weakness of the posterior and anterolateral compartments, sensory loss in the right peroneal and tibial nerve distributions, and hypoactivity of the right ankle reflex. This tumor differs from our patient whose tumor was able to be totally excised and a sural nerve graft placed, and the other difference is that our patient received both pre- and postoperative FEBRT: there was no recurrence at the extremity site of resected tumor.

Borvom, et al. [7] presented the first reported case of a leiomyosarcoma which arose from within the left sciatic nerve and involved the bifurcation of the nerve into the peroneal and tibial divisions. In this patient, the tumor, therefore, did not begin in tissue outside the nerve and invade the sciatic nerve as was the case with the patient in the publication by Benyahya, et al. and also with our patient. The site of origin of Borvon, et al.'s case was thought to be "a smooth muscle cell within the small vessel walls located outside the perineurium" [7]. The latter patient had Von Recklinghausen's disease. She presented with weakness of the left foot dorsiand plantar flexors and left leg and foot numbness. Upon resection, the extremity tumor was found to invade the epi-, pen- and endoneuria of the left sciatic nerve trunk.

\section{Sciatic nerve anatomy}

The sciatic nerve is formed from the L4-S2 spinal roots' anterior and posterior divisions and the anterior division of S3. The anterior divisions form the tibial and the posterior divisions form the peroneal nerves; the tibial and peroneal nerves join to form the sciatic nerve in the pelvis.

At the buttock level, the sciatic nerve is dorsal to the superior gemellus, obturator internus, inferior gemellus, quadratus femoris, and the adductor magnus muscles. The nerve enters the thigh deep to and between the medial and lateral hamstrings. The tumor in the case by Benyahya, et al. [6] involved the sciatic nerve at this level.

At the upper thigh level, the sciatic nerve's tibial division supplies the following muscles: the biceps femoris long head, semitendinosus, semimembranosus, and the ischial portion of the adductor magnus. The tibial division was involved by the leiomyosarcoma in the patient in the present case report. The lateral hamstring (biceps short head) is supplied by the peroneal division, which also begins in the proximal thigh. 
As the sciatic nerve reaches the junction between the middle and lower portions of the thigh, deep to the hamstrings, it bifurcates into the tibial and peroneal nerves [8]. The case by Borvom, et al. [7] had involvement of both the tibial and peroneal nerves at this bifurcation.

\section{Clinical presentation and examination}

A complete sciatic nerve lesion located proximal to the sciatic nerve's hamstring branch presents with sciatic pain and paresthesias, knee flexion difficulty, and dorsi- and plantar flexion weakness. Loss of function of the posterior tibial foot invertors, and the peroneus longus and brevis foot evertors also occurs. Sensory loss involves the posterior thigh, the lower lateral calf, and the entire foot. Delayed vasomotor changes in the involved limb present as extremity coolness, skin erythema and thinning, nail changes, and edema. If the sciatic nerve is injured below the hamstring branches and the femoral complex remains functioning, the patient can extend, lock and lift the leg, but an ankle foot orthosis (AFO) is required in order to substitute for the lack of foot dorsi- and, to a lesser degree, for plantar flexion. This brace also allows the patient to bear weight and to walk with an improved gait.

\section{Sarcoma genetics}

Sarcomas likely arise from the mesenchymal stem cell, a pluripotent cell which gives rise to varied differentiated cells [5]. When a malignant transformation of this stem cell occurs, greater than 50 histological subtypes of sarcomas result, and these have been included in the 2002 classification of the World Health Organization (WHO) [9]. Molecular classification studies have further identified distinct molecular subtypes which have refined the WHO classification [10-11].

Current molecular studies have further determined the existence of two main categories of sarcomas: 1) sarcomas with specific genetic alterations. This category is then subdivided into 1a) reciprocal translocations resulting in oncogenic fusion transcripts and $1 \mathrm{~b}$ ) specific oncogenic mutations. A second broad group is sarcomas which display 2) multiple complex karyotypic abnormalities with no specific pattern. Leiomyosarcomas fit into the second group [12]. Karyotypes in leiomyosarcomas have been found to have inconsistent numerous deletions and duplications [13].

\section{Molecular pathways involved in sarcomagenesis}

Research regarding the identification of molecular pathways involved in sarcomagenesis has made significant progress to date. Sarcoma formation involves pathway dysregulation in growth factor signaling, cell cycle progression, and cell apoptosis. Therapeutic drugs are now targeting factors in these pathways [2].

The p53 tumor suppressor pathway which is involved in cell cycle arrest and apoptosis [2] has been found to be frequently disrupted in sarcomas [2]. Specifically in leiomyosarcomas, the TP53 gene in this sarcoma subtype is commonly mutated.

The $\mathrm{Rb}$ pathway plays a regulatory role in cell cycle progression [14]. In leiomyosarcomas, the $\mathrm{RB} 1$ tumor suppressor gene, required to maintain the activation of $\mathrm{Rb}$ is often lost [2].

Receptor tyrosine kinases (RTKs) are involved in the dysregulation of cell growth and proliferation in sarcomas via aberrant signaling through these receptors. One RTK called epidermal growth factor receptor (EGFR) is overexpressed in up to 60\% of STSs. It is also involved in leiomyosarcoma formation. Another RTK often overexpressed in leiomyosarcomas is insulin-like growth factor-II (IGF-II). Activated RTKs are frequently found in sarcomas and trigger increased intracellular signaling throughout the PI3K/Akt pathway. Activation of the 
latter pathway in leiomyosarcomas may be due to a loss of the phosphatase and tensin homolog (PTEN), a negative regulator of P13K activity. Another way in which activated RTKs trigger increased intracellular signaling in sarcomas is through the Ras/Raf/MEK/MAPK pathways. Ras signaling plays a role in cell proliferation and survival as well as in cell motility and invasion. Activating Ras mutations have been found in leiomyosarcomas [14].

Vascular endothelial growth factor receptors (VEGFRs) have specific proangiogenesis effects in addition to its role in cellular proliferation; VEGFR signaling is thought necessary for angiogenesis into a growing tumor. The presence of increased amounts of this receptor protein predicted high metastatic potential and poor survival in leiomyosarcomas [15].

\section{Sarcoma invasion along peripheral nerve trunks}

Barber, et al., [16] in a very early investigation, studied 98 extremities amputated as treatment for sarcomas. They found that of the 98 cases, 38 (39\%) had grossly visible nerve involvement by various types of sarcomas. In four (11\%) of 38 extremities, a nerve was displaced by tumor, but not involved. In 13 (34\%), a nerve was adherent to the tumor, but could be stripped from it and was not grossly involved by the mass. In 11 (29\%), a nerve passed through the tumor, but retained its identity, i.e., the nerve was not enlarged or grossly involved. In six (16\%), there was localized nerve enlargement suggestive of invasion by tumor, and in four (11\%), there was diffuse enlargement of the nerve suggestive of tumor spread into and along the nerve. Thus, in the latter two categories, the gross findings suggested actual nerve involvement by sarcomas.

When the above 38 lesions were examined histologically, the 10 cases which grossly had suggested nerve involvement also had evidence of such, microscopically. An additional case was found which had not been thought to be grossly involved. Thus, a total of 11 cases were evaluated histologically. The sarcomas involved the epineurium in seven (64\%) of the 11 cases and infiltrated the epineurium and extended along the nerve in four (36\%). The perineural space is thought to have a suitable microenvironment of growth advantage, which results in tumor cell proliferation and enhancement of tumor cell spread. This layer is also thought to facilitate mechanical extension of tumors along this plane of least resistance [17].

\section{Sarcoma chemotherapy}

Local recurrence rates of $15 \%$ to $30 \%$ after treatment are the current statistics for treatment of STSs [18]. These results may be due to the fact that adult patients with STSs are treated similarly, despite great differences between STS subtypes, presentation of disease, and patient characteristics [19].

Ifosfamide and/or doxorubicin (Adriamycin) are frequently used as first line systemic therapy [19]. The patient in this case report was treated with ifosfamide and doxorubicin (Adriamycin), the latter for the first course, and then this was switched to epirubicin to allow outpatient treatment. In a study by Sleijfer, et al. [19] in which 414 patients with sarcomas were treated with ifosfamide, 660 with doxorubicin (Adriamycin) and 923 a combination of doxorubicin (Adriamycin) and ifosfamide, progression-free survival was better for ifosfamide-based therapies; however, overall survivals did not differ. Doxorubicin (Adriamycin) and ifosfamide are considered to be the most active chemotherapeutic drugs in sarcomas; ifosfamide has been shown to have a response rate of approximately $25 \%$ [19].

Gemzar (Gemcitabine) is a nucleoside antimetablite and has shown some activity in patients with advanced sarcomas. This drug was also used in our patient. A phase II trial with this drug in 29 patients, 17 (59\%) of whom had leiomyosarcomas was carried out by Okuno, et al. [20]. The following outcomes occurred; one partial response was obtained in a patient with uterine leiomyosarcoma; $83 \%$ of the 29 patients discontinued treatment due to progression and $14 \%$ 
due to toxicity or refusal.

One case report of complete disappearance of a leiomyosarcoma of the lower extremity following preoperative hyperthermia and intra-arterial doxorubicin was documented [21]. Per the publication, though, the use of intra-arterial infusion chemotherapy is not widely accepted [21].

\section{Conclusions}

Soft tissue sarcomas present a challenging disease process necessitating multidisciplinary treatment, including surgery, radiation, and chemotherapy. Though a rare entity, leiomyosarcomas are similar to other STSs in their aggressiveness, metastatic potential, and therapeutic response. As our case demonstrates, frequent metastatic surveillance, intervention, and therapy may aid in extending life-expectancy, though more studies are required to assess the quality of life, functional capability and long-term outcome.

\section{Additional Information \\ Disclosures}

Human subjects: Consent was obtained by all participants in this study. Stanford University Medical Center (SUMC) issued approval IRB \#8361. The Stanford University Medical Center (SUMC) IRB 8361 covered and covers the Stanford University Medical Center (SUMC) patients with such lesions including the operative management, outcomes and complications. The protocol has a "waiver of consent" for publications such as this one. . Conflicts of interest: In compliance with the ICMJE uniform disclosure form, all authors declare the following:

Payment/services info: All authors have declared that no financial support was received from any organization for the submitted work. Financial relationships: All authors have declared that they have no financial relationships at present or within the previous three years with any organizations that might have an interest in the submitted work. Other relationships: All authors have declared that there are no other relationships or activities that could appear to have influenced the submitted work.

\section{References}

1. Clark MA, Fisher C, Judson I, Thomas JIM: Soft-tissue sarcomas in adults. N Engl J Med . 2005, 353:701-711.

2. Demicco EG, Lazar AJ: Clinicopathologic considerations: how can we fine tune our approach to sarcoma?. Semin Oncol . 2011, 38:S3-18. 10.1053/j.seminoncol.2011.09.00

3. DeVita VJ, Hellman, S, Rosenberg, SA: Cancer: principles and practice of oncology . Lippincott Williams \& Wilkens, Philadelphia; 2001.

4. Jemal A, Siegel R, Xu J, Ward E: Cancer statistics. CA Cancer J Clin . 2010, 60:277-300.

5. Benyahya E, Etaouil N, Janani S, Bennis R, Tarfeh M, Louhalia S, Mkinsi 0: Sciatica as the first manifestation of a leiomyosarcoma of the buttock. Rev Rhum Engl Ed. 1997, 64:135-137.

6. Teicher BA: Searching for molecular targets in sarcoma. Biochem Pharmacol. 2012, 84:1-10.

7. Borvorn S, Praditphol N, Nakornchai V: Leiomyosarcoma in peripheral nerve: the first case report. J Med Assoc Thai . 2003, 86:1080-1085.

8. Kim DH, Murovic JA, Tiel R, Kline DG: Management and outcomes in 353 surgically treated sciatic nerve lesions. J Neurosurg. 2004, 101:8-17.

9. Fletcher C, Unni, KK, Mertens F: Pathology and Genetics of Tumours of Soft Tissue and Bone Lyon. IARC Press, 2002.

10. Corless CL, Schroeder A, Griffith D, Town A, McGreevey L, Harrell P, Shiraga S, Bainbridge T, Morich J, Heinrich MC: PDGFRA mutations in gastrointestinal stromal tumors: frequency, spectrum and in vitro sensitivity to imatinib. J Clin Oncol. 2005, 23:5357-5364.

11. Ducimetiere F, Lurkin A, Ranchere-Vince D, Decouvelaere AV, Peoc'h M, Istier L, Chalabreysse P, Muller C, Alberti L, Bringuier PP, Scoazec JY, Schott AM, Bergeron C, Cellier 
D, Blay JY, Ray-Coquard I: Incidence of sarcoma histotypes and molecular subtypes in a prospective epidemiological study with central pathology review and molecular testing. PLoS One . 2011, 6 :e20294.

12. Skubitz KM, Pambuccian S, Manivel JC, Skubitz AP : Identification of heterogeneity among soft tissue sarcomas by gene expression profiles from different tumors. J Transl Med . 2008, $6: 23$.

13. de Alava E: Molecular pathology in sarcomas . Clin Transl Oncol. 2007, 9:130-144.

14. Mahalingam D, Mita A, Sanldiala K, Swords R, Kelly K, Giles F, Mita MM: Targeting sarcomas: novel biological agents and future perspectives. Curr Drug Targets. 2009, 10:937-949.

15. Potti A, Ganti AK, Tendulkar K, Sholes K, Chitajallu S, Koch M, Kargas S:: Determination of vascular endothelial growth factor (VEGF) overexpression in soft tissue sarcomas and the role of overexpression in leiomyosarcoma. J Cancer Res Clin Oncol. 2004, 130:52-56.

16. Barber JR, Coventry MB, McDonald JR: The spread of soft-tissue sarcomata of the extremities along peripheral-nerve trunks. J Bone Joint Surg Am. 1957, 39-A:534-540.

17. Bockman DE, Buchler M, Beger HG: Interaction of pancreatic ductal carcinoma with nerves leads to nerve damage. Gastroenterology . 1994, 107:219-230.

18. Mack LA, Crowe PJ, Yang JL, Schachar NS, Morris DG, Kurien EC, Temple CL, Lindsay RL, Magi E, DeHaas WG, Temple WJ: Preoperative chemoradiotherapy (modified Eilber protocol) provides maximum local control and minimal morbidity in patients with soft tissue sarcoma. Ann Surg Oncol. 2005, 12:646-653.

19. Sleijfer S, Ouali M, van Glabbeke M, Krarup-Hansen A, Rodenhuis S, Le Cesne A, Hogendoorn PC, Verweij J, Blay JY. : Prognostic and predictive factors for outcome to first-line ifosfamidecontaining chemotherapy for adult patients with advanced soft tissue sarcomas: an exploratory, retrospective analysis on large series from the European Organization for Research and Treatment of Cancer-Soft Tissue and Bone Sarcoma Group (EORTC-STBSG). Eur J Cancer. 2010, 46:72-83. 10.1016/j.ejca.2009.09.02

20. Okuno S, Edmonson J, Mahoney M, Buckner JC, Frytak S, Galanis E: : Phase II trial of gemcitabine in advanced sarcomas. Cancer. 2002, 94:3225-3229.

21. Khalek Y, Vilor M, Sorrentino J, Brown M, Wills J, Herrera L: Complete disappearance of a leiomyosarcoma of the lower extremity following preoperative hyperthermia and intraarterial doxorubicin.. J Surg Oncol. 1993, 52:272-275. 